\section{But of course . . .}

Roger Pynn

Fractals. By Jens Feder. Plenum: 1988. Pp. 283. \$49.50.

IN reading this book, I kept wondering for whom it had been intended. Not for the idly curious who are searching for an intuitive grasp of the concept of fractals: Benoit Mandelbrot's The Fractal Geometry of Nature provides that so much more elegantly. Nor for the mathematician, who can be left with no lingering doubts: although I cannot direct such purists, I know that Feder's book would not satisfy them. Finally, it dawned. If I were teaching a course on fractals to any group of natural scientists, this would be the course book Of course, such is the route that Professor Feder has followed, and had I paid more attention to his preface I would have realized as much.

With the exception of the chapter on multifractals, which is confusing and undirected, the book is pedagogically sound. It gathers together in one place the various definitions of fractal dimension which are used in analysing experimental data and provides ample references to their use. The chapter on percolation is a clear discussion of this phenomenon and its relation to fractal concepts, and includes several new results which have helped to unify the subject. I particularly enjoyed the account of fractal records in time and rescaled-range analysis, which $I$ have not previously seen explained with such clarity. The link between this subject and studies of brownian motion is well described, and is illustrated by a series of graphs and pictures obtained from simulations programmed on a personal computer. Essential differences between self-similarity and self-affinity are dealt with approachably and succinctly. Several algorithms for the generation of fractal surfaces, landscapes and clouds are discussed, but Feder's book is intended to introduce this topic rather than to celebrate a new art form.

A great number of the examples used in the book would make excellent research or teaching projects for students. Perhaps, indeed, that is how they were born. The means required to study these examples are modest: a PC, a few purchases at a local hardware store and access to a public library would probably allow an interested student to generate enough data for another monograph within a year. There are, too, lots of references to the recent literature, providing the reader with a window on more than 200 other works.

Like Feder, I believe that Mandelbrot's concept of fractal geometry provides a sturdy framework for the analysis of natural phenomena in diverse fields of science. If that view continues to spread, it will not be long before the study of fractals is a mandatory part of university curricula. The book to support such a course is already available. This is it.

Roger Pynn is at the Los Alamos National Laboratory, MS H805, Los Alamos, New Mexico 87545, USA

\section{Modern virtues}

\author{
Richard S. Westfall
}

The How and the Why: An Essay on the Origins and Development of Physical Theory. By David Park. Princeton University Press: 1988. Pp.459. \$35.

WELL into The How and the Why, I found that I was becoming annoyed with it. The subtitle appears to offer a book in the history of physics (or of physical theory), and the contents, beginning with the Greeks and proceeding chronologically through antiquity, the Middle Ages, and the scientific revolution towards contemporary physics, confirm that impression.

In all honesty, I was not greatly impressed. The chapters on the Greeks are disjointed collections of short essays about individual philosophers. There is no coherent account of the considerable mediaeval enterprise in natural philosophy. When I got to the scientific revolution, the period in which I claim some expertise, I found numerous errors, and my ill humour was by that time spilling over
More than popularization, however, Park's book is also aimed at philosophically inclined physicists who want to stand back for a moment and contemplate the forest instead of the trees. In this respect The How and the Why considers not so much the content of contemporary physics as the nature of physical theory. I cannot claim expertise in modern physics, and the definitive word on Park's book will have to be delivered by others. But I find it difficult to believe that they will not be favourably impressed.

I began with words of criticism, so I owe it to candour to indicate briefly their foundation. I confine myself to Newton. Park asserts that "no letter $t$ or its equivalent ever appears [in the Principia]. The only numbers in the Principia relate to distances; everything about time is implicit" (p. 232). I invite him to look again at Prop. VI, which enters into the argument of most of the rest of Book I. A bit later (p. 248) Park appears to state that Newton's work contains only geometrical diagrams and the waving of hands but no proofs. I have no idea what he can mean by those words, but it is hard to believe that anyone who says as much has seriously studied the Principia. I could multiply instances concerned with Newton and other figures of the scientific revolution at some length, and in a book that offers itself as an essay on the origins and development of physical theory, this constitutes a considerable fault. But I have made the point and wish to go no further. Everything in the book builds toward the final third, and one should not obscure its achievement by cavilling over subordinate matters.

In the climactic section of The How and the Why, extended accounts of relativity and quantum mechanics and of the evidence on which they rest lead on to the most recent endeavours in physical theory, such as superstrings, and to related work in cosmology. Throughout, Park concentrates on general themes, such as simplicity, symmetry and determinism, which were also central to his historical account of physical theory. One who is not a physicist cannot comment definitively on these chapters, and beyond the inherent complexity of the subject is Park's inclusion of the latest developments in contemporary physical theory, which will be new to the vast majority who are not themselves theoretical physicists. I am not a complete stranger to literature of this genre, and wish to say that I have never encountered more enlightening explications of these difficult issues. I wish Park had got the historical details a bit more straight. But I happily settle for the extraordinarily lucid and penetrating analyses of contemporary physical theory.

Richard S. Westfall is a Professor in the Department of History and Philosophy of Science, 130 Goodbody Hall, Indiana University, Bloomington, Indiana 47401, USA. 